\title{
Thermal properties and Fisher information for diatomic molecular Hellman-modified-Kratzer potential model
}

\author{
Akpan Ikot ${ }^{1}$, Uduakobong Okorie ${ }^{2}$, Gaotsiwe Joel Rampho ${ }^{1}$, Collins Edet ${ }^{3}$, Precious \\ Amadi ${ }^{4}$, Ita Akpan ${ }^{5}$, Ridha Horchani ${ }^{6}$, and Hewa Abdullah ${ }^{7}$ \\ ${ }^{1}$ University of South Africa - Florida Campus \\ ${ }^{2}$ Akwa Ibom State University \\ ${ }^{3}$ Federal University of Technology Minna \\ ${ }^{4}$ University of Port Harcourt \\ ${ }^{5}$ University of Calabar \\ ${ }^{6}$ Sultan Qaboos University \\ ${ }^{7}$ Salahaddin University- Hawler
}

June 15, 2020

\begin{abstract}
In this paper, we solved the Schrodinger equation with Hellmann-modified Kratzer potential using Nikiforov-Uvarov-Functional Analysis (NUFA) method. The obtained energy is used to study the numerical results of the ro-vibrational energy spectra for some selected diatomic molecules and their thermodynamic properties. In addition, we also investigated the Fisher information for three diatomic molecules and they all satisfied the Stam-Cramer-Rao inequalities uncertainty relations. Special cases of the potential are discussed and we compute the numerical eigenvalue of the modified Kratzer, Kratzer-Feus and Hellmann potentials for comparison with other analytical methods. The results of the present study agree with the results obtained with other known methods.
\end{abstract}

\section{Hosted file}

Updated NUFA method for Hellman potential @ Ikot.docx available at https://authorea.com/ users/291200/articles/459606-thermal-properties-and-fisher-information-for-diatomicmolecular-hellman-modified-kratzer-potential-model 